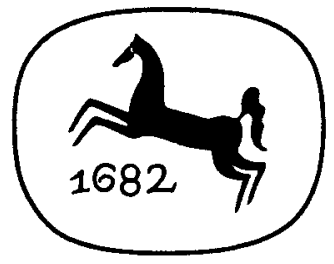

KLASSIK UND Moderne 


\title{
Klassik und Moderne
}

\author{
Die Weimarer Klassik \\ als historisches Ereignis \\ und Herausforderung \\ im kulturgeschichtlichen Prozeß \\ Herausgegeben \\ von Karl Richter und Jörg Schönert
}

Walter Müller-Seidel zum

65. Geburtstag

J. B. Metzlersche

Verlagsbuchhandlung

Stuttgart 
CIP-Kurztitelaufnahme der Deutschen Bibliothek

Klassik und Moderne : $\mathrm{d}$. Weimarer Klassik als histor. Ereignis u. Herausforderung im kulturgeschichtl.

Prozeß ; W. Müller-Seidel zum 65. Geburtstag / hrsg. von Karl Richter u. Jörg Schönert. - Stuttgart :

Metzler, 1983.

ISBN 978-3-476-00534-2

NE: Richter, Karl [Hrsg.]; Müller-Seidel, Walter:

Festschrift

ISBN 978-3-476-00534-2

ISBN 978-3-476-03181-5 (eBook)

DOI 10.1007/978-3-476-03181-5

(C) 1983 Springer-Verlag GmbH Deutschland

Ursprünglich erschienen bei J. B. Metzlersche Verlagsbuchhandlung und Carl Ernst Poeschel Verlag GmbH in Stuttgart 1983 


\section{Inhalt}

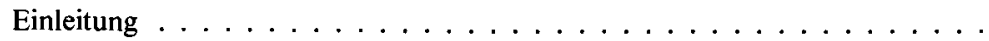

TEIL I

Perspektiven und Probleme des KLassik-Begriffs

Herbert JAUMANN: Vom »klassischen Nationalautor« zum »negativen Classiker«. Wandel literaturgesellschaftlicher Institutionen und Wirkungsgeschichte, am Beispiel Wieland.

MANFRed BeETZ: "In den Geist der Alten einzudringen «. Altphilologische Hermeneutik als Erkenntnis- und Bildungsinstrument der Weimarer Klassik. . . .

DieTER BORCHMEYER: Rhetorische und ästhetische Revolutionskritik: Edmund Burke und Schiller. . . . . . . . . . . . . . . . . .

Volker HoffmanN: Elisa und Robert oder das Weib und der Mann, wie sie sein sollten. Anmerkungen zur Geschlechtercharakteristik der Goethezeit. . . . . .

Michael Titzmann: Probleme des Epochenbegriffs in der Literaturgeschichtsschreibung. . . . . . . . . . . . . . . .

\section{TEIL II}

Die Weimarer Klassik im historischen Kontext DER >GoethezeIT<

WaLter Pache: Idylle und Utopie: Zur Rezeption Oliver Goldsmiths in der Goethezeit. . . . . . . . . . . . . . . . .

Wolfgang STauch-v. Quitzow: Ein Lustspiel auf dem Wege zur Klassik? Goethes Die Mitschuldigen: Vom Theaterexperiment zum Weimarer Bühnen-

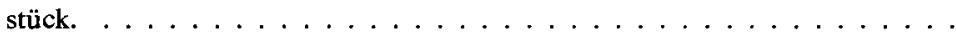

URSUla SegEBrecht: Götter, Helden und Goethe. Zur Geschichtsdeutung in Goethes Iphigenie auf Tauris. . . . . . . . . . . . . . . . . . . . .

WULF SEgEBRECHT: Naturphänomen und Kunstidee. Goethe und Schiller in ihrer Zusammenarbeit als Balladendichter, dargestellt am Beispiel der Kraniche des Ibykus. . . . . . . . . . . . . . . . . . . .

KLAUS H. KIEFER: Okkultismus und Aufklärung aus medienkritischer Sicht. Zur Cagliostro-Rezeption Goethes und Schillers im zeitgenössischen Kontext. . . .

Thomas E. Bourke: Vorsehung und Katastrophe. Voltaires Poème sur le désastre de Lisbonne und Kleists Erdbeben in Chili. . . . . . . . . . . . . . . . . .

Gregor Thurmair: Das Gesetz der Dichtung. Hölderlins Rezeption der griechischen Poesie. . . . . . . . . . . . . . . . . . . .

Gisela Henckmann: "... wie die alten Amazonen der Fabelwelt«. Die antike Mythologie im Werk Achim von Arnims. . . . . . . . . . . . .

GUNTER Hess: Goethe in München. Literarische Aspekte der Geschichte und Wirkung von Stielers Dichter-Porträt. . . . . . . . . . . . . 


\section{TEIL III}

Funktion und Bedeutung der Weimarer KLassik fÜr Das BewusstSEIN DER MODERNE

ADALBERT WiCHERT: Bismarck und Goethe. Klassikrezeption der deutschen Geschichtswissenschaft zwischen Kaiserreich und Drittem Reich. . . . . . . . . .

Horst ThомE: Goethe-Stilisierung bei Sigmund Freud. Zur Funktion der enigmatischen Persönlichkeit in der psychoanalytischen Bewegung. . . . . . . . .

Michael Stark: „Uns ist die Klassik ein Muster ohne Wert. «Zur expressionistischen Provokation der autoritären Aneignung von Tradition. . . . . . . . .

MaRIanNE WÜNSCH: Das Modell der "Wiedergeburt « zu "neuem Leben « in erzählender Literatur $1890-1930$. . . . . . . . . . . . . . . . . . . .

Thomas Anz: Der schöne und der häßliche Tod. Klassische und moderne Normen literarischer Diskurse über den Tod. . . . . . . . . . . . . . . . . . .

KARL RICHTER: Emotionalisierung durch Sachlichkeit. Ein wirkungsästhetisches Paradigma der Moderne.

\section{TEIL IV}

\section{Die Weimarer Klassik im kulturellen Leben der Gegenwart}

MaximiLian NutZ: Restauration und Zukunft des Humanen. Zur westdeutschen Goethe-Rezeption von 1945 bis 1949 . . . . . . . . . . . . . . . . . . .

WALTER Gebhard: Im Streit um die Klassik. Anmerkungen zur didaktischen Diskussion über die Antiquiertheit klassischer Literatur.

IRMGARD ACKERMANN: Die Weimarer Klassik im Schullesebuch. . . . . . . . . 504

ERNST WEBER: Transkriptionen: Goethes Romane in der Literatur der siebziger Jahre. . . . . . . . . . . . . . . . . . . . .

JÜRGEN G. SANG : Erwerb eines Klassikererbes? Rezeptionshaltungen bei Hacks und Handke . . . . . . . . . . . . . . . . . . . . . . . .

Jörg SCHÖNERT: Der »Geheime Rat « und die angefochtene Existenz der Dichter. Goethe als Gegenbild im biographischen Erzählen der neueren DDR-Literatur.

GunTER ReIss: Dramaturgie der Gewalt. Das Verhör als kommunikative Figur in der Geschichte und im Drama des 20. Jahrhunderts. . . . . . . . . . . .

Gerd SAUTERMEISTER: Aufklärung und Körpersprache. Schillers Drama auf dem Theaterheute. . . . . . . . . . . . . . . . . 618

Mitarbeiter des Bandes . . . . . . . . . . . . . . . . . . . . 641

Personenregister . . . . . . . . . . . . . . . . . . . . 647 


\section{Einleitung}

1.

,Klassik und Moderne - der eindeutige Gegensatz, der diese Formel im epochengeschichtlichen und poetologischen Verständnis prägt, ist zu differenzieren, wenn das Begriffspaar in eine weiterreichende historische Perspektive gestellt wird und man seine Entstehungs- und Wirkungsgeschichte in ihren Bedingungen und Folgen untersucht[1]. In diesem Band sind erste geschichtliche Festlegungen damit vollzogen, daß wir von der >Weimarer Klassik « sprechen und die Rolle dieses >kulturellen Paradigmas im Kulturwandel der >jüngeren Moderne ‘ [2] und der Gegenwart, also etwa ab 1890 verfolgen [3]. Damit soll zunächst ausgeschlossen sein, das Verhältnis von >klassisch` und >modern` entwicklungstypologisch zu verstehen - etwa als wiederkehrenden Konflikt in der Kunstevolution, als notwendige Opposition zwischen Traditionsbezug und Traditionsbruch [4]. Der thematische Zusammenhang der hier vorgelegten Studien verweist darauf, daß Literatur-, Kultur- und Gesellschaftsgeschichte aufeinander zu beziehen sind. Dieses Wechselverhältnis wäre theoretisch genauer zu begründen und in seinen untersuchungspraktischen Konsequenzen darzustellen. Ein solches Unternehmen konnte freilich nicht das Ziel einer Publikation sein, die Beiträge von 28 Autoren vereint. Die gemeinsame Orientierung der Aufsätze liegt darin, daß weder >Klassik « noch > Moderne` als Fixpunkt für normative Erwartungen in einem geschichtsoptimistischen oder kulturpessimistischen Entwicklungsmodell eingesetzt werden. Sie gelten uns als historische Konstellationen, die in ihrer jeweiligen kulturellen Bedeutung und gegenseitigen Zuordnung im geschichtlichen Prozeß unterschiedliche Wertungen erfahren können und erfahren haben.

Wir verstehen , Weimarer Klassik a als Kulturprogramm, das sich in den literarischen, publizistischen und wissenschaftlichen Aktivitäten verwirklicht, die mit den Namen von Goethe und Schiller verbunden sind. Als Eingrenzung wäre der Zeitraum von 1786 bis etwa 1808 zu wählen, zwischen Goethes Reise nach Italien und den ersten Texten, die seinem Alterswerk zugerechnet werden - etwa Die Wahlverwandtschaften und Pandora. Darin markieren die Jahre von 1794 bis 1805 mit der Zusammenarbeit der beiden `Klassiker` die Kernzone des \Klassischen<. In kulturgeschichtlicher Sicht muß das Ereignis ,Weimarer Klassik« mit der Ausbildung eines modernen Gesellschaftssystems im deutschen Territorialbereich verbunden werden. Für diese Phase hat die sozialhistorische Forschung mit dem Ausgangspunkt $>1770$ < ein weithin akzeptiertes Grenzdatum vorgeschlagen, und vielfach stimmt man auch darin überein, daß dann in den Jahren um 1830 neue und beschleunigte Entwicklungen einsetzen. Die Periode 1770-1830 bildet in Teil I und II unseres Bandes den zeitlichen Rahmen für die Diskussion der Weimarer Klassik. Literarhistorisch bezeich- 
nen wir sie mit dem Hilfsbegriff ` Goethezeit $`$ Geht man von einer umfassenden gesellschaftsgeschichtlichen Perspektive aus, dann erscheint das kulturelle $\mathrm{Pa}-$ radigma der Weimarer Klassik als geschichtsmächtiger Faktor im Prozeß einer sich konstituierenden Moderne - als programmatische Antwort auf Vorgänge und Ereignisse, die für uns zwar > Historie ‘ geworden sind, in ihrer Wirkungsgeschichte und mit ihrem Problempotential jedoch in das Hier und Heute hineinreichen. Damit rücken wir `Klassik « in das Spannungsfeld zweier unterschiedlich begründeter Begriffe von $>$ Moderner.

Im literarhistorischen Gebrauch von Moderne werden vor allem literarische Entwicklungen und neue Konstellationen des literarischen Lebens angesprochen, die sich zum Ende des 19. Jahrhunderts abzeichnen und bis in die Gegenwart bestimmend sind. Diese Kategorie der Moderne ist von der geschichtlichen Situation um 1900 geprägt, vom Selbstverständnis wichtiger Schriftsteller dieser Zeit und den Einordnungen der Literaturwissenschaft, die daran anschließen. Mit der Positionsbestimmung 'modern` sollten Abgrenzungen zur Literatur des 19. Jahrhunderts und zu gleichzeitigen traditionalistischen Einstellungen getroffen werden. Setzt man für den Zeitraum von 1890 bis etwa 1930 die Epochenbezeichnung >Moderne - oder besser jjüngere Moderne ‘ ein, dann ergeben sich ähnliche Probleme wie bei der Verwendung von $>$ Klassik` für die literaturgeschichtliche Periode des späten 18. und frühen 19. Jahrhunderts. Während sich der Klassik-Begriff noch mit `Weimarer Klassik ‘ präzisieren und eingrenzen läßt, erweisen sich ähnliche Versuche für >Moderne〈 als untauglich. Wir beziehen uns deshalb hier mit sjüngere Moderne` auf den Abschnitt 1890-1930 als ein Ganzes. Die Zuordnung entspricht in ihrer Reichweite und ihrem terminologischen Status etwa einem Begriff wie `Goethezeit<. ,Jüngere Moderner umschließt sowohl mehrere kulturelle Paradigmen (beispielsweise `Das junge Wien $`$ ) und Literaturprogramme (etwa Neuromantik, Expressionismus, Neue Sachlichkeit usf.) als auch Einstellungen, die sich an Norm und Wirkungsgeschichte der Weimarer Klassik im 19. Jahrhundert orientieren. Freilich wachsen die Schwierigkeiten im Gebrauch von >Moderne`, je größer die zeitliche Distanz zu jener Periode wird, die diese Kennzeichnung für sich beanspruchte. Prägungen wie $>$ Postmoderne weisen darauf hin, daß auch die >Modernität ` von Kunst und Literatur im beginnenden 20. Jahrhundert als historisches Faktum zu verstehen ist.

Im Umgang mit dem Moderne-Begriff entstehen in literaturgeschichtlichen Darstellungen vielfach dann Mißverständnisse, wenn 'klassisch ‘ und 'modern ‘ in poetologischer Sicht gebraucht und aufeinander bezogen sind. Dabei werden zumeist einzelne Perspektiven in den Kunstauffassungen der Weimarer Klassik und in den literarischen Aufbruchsbewegungen um 1900 isoliert und normativ gesetzt - beispielsweise Traditionsbezug gegen Traditionsbruch. Oft führen Überlagerungen von historischer und normativer Verwendung der Begriffe dazu, daß rals Literaturgeschichter etwa nur die Ablösung >des Klassischen` durch `das Moderne` verfolgt und als ein kontinuierlicher Prozeß beschrieben wird. 
Um für solche Probleme der Literaturgeschichtsschreibung eine-gleichsam neutrale - Bezugsinstanz zu haben, benutzen wir auch den gesellschaftsgeschichtlichen Begriff der Moderne. Er entspricht dem Gegenstandsbereich dieses Bandes und umfaßt den Zeitraum vom Ausgang des 18. Jahrhunderts bis zur Gegenwart. In diesem Rahmen können die Implikationen gegenwärtiger literaturgeschichtlicher Periodenbildungen ebenso diskutiert werden wie die Voraussetzungen und Wirkungen historischer Abgrenzungen und Selbstdefinitionen. Auch die verschiedenen Vorgänge, in denen die Weimarer Klassik normativen Charakter erhält, aktualisiert oder kritisch distanziert wird, erscheinen dann als geschichtlich zu verstehende Phänomene. Unser Titel Klassik und Moderne verweist also auf Entstehung und Funktion des kulturellen Paradigmas ,Weimarer Klassikı in den geschichtlichen Entwicklungen der zurückliegenden zwei Jahrhunderte: auf die historische Rolle der Klassik in der Moderne.

Dabei ist dem möglichen Mißverständnis vorzubeugen, daß hier Gesellschaftsgeschichte und kultureller Prozeß, insbesondere die Geschichte der Literatur, in eine spannungslose Parallelität gesetzt werden sollen. Zum einen verläuft die Ausbildung einer smodernen Gesellschaft ‘ im deutschen Sprachraum weder einheitlich noch kontinuierlich; hier muß regional differenziert und mit der 'Gleichzeitigkeit des Ungleichzeitigen، gerechnet werden. Zum anderen kann es als ein Charakteristikum dieses Gesellschaftstyps angesehen werden, daß der kulturelle Handlungsbereich als relativ selbständiger Aktions- und Erfahrungsraum sausdifferenziert « wird. Die deutsche Entwicklung ist dadurch gekennzeichnet, daß die kulturellen Prozesse im letzten Drittel des 18. Jahrhunderts in einem besonders raschen Tempo verlaufen, daß der Kulturwandel den politischen, sozialen und ökonomischen Veränderungen svorauseilt‘. Damit ließe sich die Situation zwischen 1770 und 1830 als Spannungsverhältnis zwischen dem kulturellen und dem sozio-ökonomischen Wandel beschreiben. Beide Prozesse unterscheiden sich sowohl in ihrer Dynamik als auch in ihren Zielen. Die Divergenz wird in der Zeit durchaus bewußt. Dies hat zur Folge, daß kulturelles und gesellschaftliches Geschehen voneinander abgelöst werden sollen und Literatur als Erfahrungswelt mit eigenen Gesetzen angesehen wird. Aus der gestörten Homologie der Prozesse ergibt sich aber auch die Möglichkeit, in den kulturellen Diskursen die gesellschaftlichen Vorgänge kritisch zu begleiten und zu deuten. Was die kulturelle Reflexion sozialer Realität leisten kann, wird allerdings vielfach überschätzt. So kommt es beispielsweise beim Anspruch, literarisch >Aufklärung zu befördern, nach 1780 zu krisenhaften Erfahrungen. Die > Pathologien der Moderne hinterlassen von Anfang an auch in der Kulturgeschichte ihre Spuren.

Die relative Autonomie des Kulturprozesses führt nicht nur dazu, daß etwa in einer vorauseilenden Kulturrevolution Triebkräfte der gesellschaftlichen Entwicklung freigesetzt werden, zum Beispiel im Entwurf von Gesellschaftsutopien, Bildungs- und Erziehungsprogrammen [5]. Ebenso können bestimmte kulturelle Konstellationen soziale Wandlungsprozesse hemmen und 'falsches Bewußtsein ‘ von der Wirklichkeit gesellschaftlicher Verhältnisse vermitteln. 
Für die frühe Periode in der Entwicklung einer modernen Gesellschaft in Deutschland ist charakteristisch, daß für Kunst und Literatur die Funktionen im gesellschaftlichen Prozeß in wechselnden und kontroversen Entwürfen neu bestimmt werden. Es sei der Versuch gewagt, die Weimarer Klassik in dieser historischen Perspektive so einzuordnen, daß ihr Programm das Vorauseilen des Kulturwandels, der revolutionäre Züge zeigt, verlangsamt und zu kontrollieren versucht. Durch den Anschluß an Tradition und die Forderungen nach >schönem Maß< und shumanem Ethos` werden Grenzen gesetzt, sollen kultureller und gesellschaftlicher Prozeß unter den Bedingungen des Möglichen einander angenähert werden. Für die Weimarer Klassik erscheint das Auseinanderklaffen von Anspruch (dem denkbaren Zustand des befreiten Menschengeschlechts) und Realität (dem Beharrungsvermögen des Absolutismus) als Katastrophe im Schreckbild einer sozialen Revolution, die in kultureller Reflexion nicht mehr zu erfassen ist. Damit widersetzt sich das Kulturprogramm von Weimar nicht einer >Entwicklung zur Moderne ( [6], sondern es dringt - im Gegensatz zum Selbstverständnis der entschieden aufklärerischen Autoren oder der Protestliteratur des `Sturm und Drang - auf Koordination von kulturellem und gesellschaftlichem Wandel. Angesichts der wachsenden Differenzierung der gesellschaftlichen Handlungsbereiche werden rentdifferenzierende، Prozesse eingeleitet und befördert. Die Probleme, die sich bei Ausbildung und Durchsetzung der modernen Gesellschaft - mit allen ihren Begleiterscheinungen - ergeben, bleiben jedoch als Bezugspunkte in den Problemlösungen aufbewahrt, die von der Weimarer Klassik literarisch entwickelt werden. In der Wirkungsgeschichte können sie in unterschiedlicher Weise aktiviert oder verdrängt werden.

Ebenso wie der Entstehung der Weimarer Klassik ist auch ihrer Wirkungsgeschichte nicht nur unter den Aspekten der Stoff-, Motiv- und Formtraditionen oder im Bereich ästhetischer Entwürfe und Kunstdiskussionen nachzugehen. Wichtig sind die unterschiedlichen öffentlichen Diskurse, in denen Anspruch und Stellenwert des kulturellen Paradigmas im historischen Prozeß geprüft und festgelegt werden. Die Kritik an prinzipiellen Tendenzen der Wirkungsgeschichte muß sich jedoch auf ein historisch kontrolliertes Bild vom bewirkenden Faktor, der Weimarer Klassik, beziehen. Es wird auch seinerseits nicht objektive Gültigkeit beanspruchen, aber es kann mit der skizzierten gesellschaftsgeschichtlichen Perspektive von 'Klassik in der Moderne halten und weiterführende Fragen auslösen. Dabei gilt es, die reichen Ergebnisse literaturgeschichtlicher Forschung kritisch zu vergleichen und daran anzuschließen. 
2.

Bei einer Korrektur des Klassik-Bildes, das sich in der Literaturgeschichtsschreibung des 19 . Jahrhunderts ausgeprägt hat, ist zu betonen, daß mit dem Kulturprogramm der Weimarer Klassik nur eine - wenn auch folgenreiche Position in der Literaturgeschichte des ausgehenden 18. Jahrhunderts erfaßt wird. Ebenso müssen in der Biographie der beiden Klassiker >vorklassischer Kunstauffassungen beachtet werden und bei Goethe zudem eine >nachklassische Phase. Sie zeichnet sich - unterschiedlich akzentuiert - in den Werken ab, die nach 1808 erschienen. Das Fatale an solchen Einordnungen liegt in der Ter-

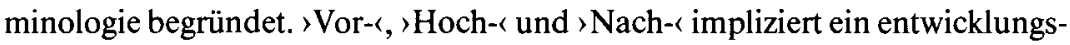
logisches Verständnis, das den geschichtlichen Sachverhalt verfehlt. Das sklassische Kunstprogramm، hat höchst unterschiedliche Impulse aufgenommen. $\mathrm{Zu}$ verknüpfen ist es mit Goethes Hinwendung zur Kunst der Antike, zur >Lebenskultur` Italiens und zum Modell biologischer Evolution. Es wird abgesichert durch Schillers Geschichtsphilosophie und Anthropologie und präzisiert sich etwa ab 1792-94 in der kritischen Distanz zu den politischen Erfahrungen, die sich für beide Autoren in diesen Jahren mit der Französischen Revolution verbinden [7]. Zugleich bezeichnet es eine deutliche Zäsur in der literarischen Biographie Goethes und Schillers. Im Entwurf einer neuen Kunst werden auch neue Antworten auf Zeiterfahrungen gesucht, die sich nicht grundsätzlich verändert haben, sondern belastender und gefährdender geworden sind. Sie schlieBen vor allem die Probleme ein, die sich aus dem Umbruch in der Gesellschaftsordnung, aus den veränderten Funktionen von Philosophie und Religion, den neuen Verhältnissen von Mensch und Natur, von Technik und Kultur, von Individuum und Gesellschaft ergeben. Von der Weimarer Klassik werden Begrif-

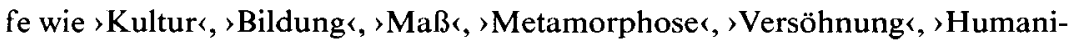
tät ‘ als zentrale Größen in ein Konzept von Evolution eingebracht, das den Aufbruch in die Moderne als Erneuerung verstehen will. Gegründet ist es auf die durchdachte Aneignung von Tradition und auf behutsame Innovationen. Es erscheint als »gegenrevolutionäres Kulturprogramm«[8], als Entwurf eines Gesellschaftswandels, der im Zeichen von Geist und Kultur - anstelle von Macht und Gewalt - steht.

Der Kulturbegriff (und ihm zugeordnet die Bildungsidee) bezieht das neu interpretierte aufklärerische Orientierungsmodell > Natur auf den sozialen Erfahrungsbereich, die skünstliche` Ordnung des menschlichen Zusammenlebens. In einer kulturell verantworteten und reflektierten Evolution sollen Grundlagen für einen Wandel in den gesellschaftlichen Verhältnissen geschaffen werden, der von der Bildung des Individuums zu einer harmonisch entwikkelten >Persönlichkeit ‘ abhängig ist. Voraussetzung dafür ist die planvolle und allgemeine Aneignung des Kunsterbes der 'klassischen، Antike. Die Kunstprinzipien und Kunstnormen dieser neuen Kultur sind jedoch nicht mit der Autorität von Lehrmeinungen und Schulen durchzusetzen, sondern im philosophischen Diskurs der Ästhetik zu begründen. Dazu entwickelt die idealistische 
Ästhetik um 1800 die Vorstellung vom rautonomen Kunstwerk ‘. In ihm können real widerstreitende Kräfte symbolisch im 'freien` Zusammenspiel versöhnt werden, indem Erfahrungswirklichkeit in den sschönen Schein ‘ der Kunst verwandelt wird. Das Kunstwerk steht zugleich als Bild für die Harmonie von Sinnlichkeit und Verstand im vollkommenen Menschen und für den Ausgleich widerstrebender Interessen im vollkommenen Staatswesen [9]. Daß philosophische Entwürfe und die Bilder der künstlerischen Phantasie der gesellschaftlichen Realität gerade in den letzten Jahrzehnten des 18. Jahrhunderts immer mehr vorauseilten oder als fremd erschienen, wurde vielfach schmerzlich empfunden und als verstörend erlebt. Im Kunstprogramm der Weimarer Klassik gelten solche Divergenzen dagegen als Notwendigkeiten in einem Erziehungsplan, der sich im - freilich ungeschichtlichen - Verständnis von Kunst und Kultur der griechischen Antike rechtfertigt. ) Fremd ist das Vergessene, das nur neu entdeckt und angeeignet werden muß. Der geschichtliche Prozeß vermag sich nicht nur im Blick auf eine neue Zukunft zu versichern, sondern auch in der Erneuerung von Tradition. In der Literatur sollen der Impetus der aufklärerischen Kritik, die literarische Selbstvergewisserung über Intensität und Reichweite der Gefühle sowie die grenzüberschreitende Dynamik einer freigesetzten >Einbildungskraft $\triangleleft$ zügelnd und mäßigend auf die Prinzipien ’natürlicher Ordnungen und einer schrittweisen Entwicklung bezogen werden. Evolution vollzieht sich, wenn Gegensätze auf einer höheren Ebene der Imagination und Reflexion überwunden sind [10] - gestützt auf die leitende Idee des Guten, Wahren und Schönen. Diese Idee ist ihrerseits eine Kunstleistung, indem sie ein bestimmtes Bild der Vergangenheit (der Antike) mit dem Entwurf zur Zukunft eines neuen Menschengeschlechts verbindet.

Doch ist die Forderung , Ganzheit< als künstlerischer und lebenspraktischer Leitwert durchaus gegen die konkreten gesellschaftlichen Erfahrungen der Situation um 1800 gerichtet. Die moderne Entwicklung zur Arbeitsteiligkeit und zum Spezialistentum wird als Gefahr gesehen, daß Leben verkümmert und Wissen zerfällt. Aus dem sprachlichen Potential und der Anschauungsfülle einer neuen Literatur soll auch die Möglichkeit erwachsen, literarische - und andere kulturelle - Diskurse auf neue Weise mit den Wissenschaften in Beziehung zu bringen (vor allem mit Philosophie, Naturwissenschaft und Historiographie). Voraussetzung dazu ist eine bewegliche Sprache, die nicht in ein enges Korsett grammatischer Regeln oder rhetorischer Muster eingezwängt ist und sowohl die intellektuelle Neugier wie das emotionale Ausdrucksverlangen des sich selbst definierenden Subjekts zu tragen vermag. Eine solche Sprachkompetenz gilt es `literarisch< auszubilden. Damit werden Wege erschlossen, um - in einem neuen Universalismus - solche Strukturen zu erfassen, die scheinbar getrennte Lebens- und Erfahrungsbereiche miteinander verknüpfen, um - des weiteren schwierige Wissenszusammenhänge ranschaulich ‘ zu vermitteln sowie komplexe Wahrnehmungs- und Deutungsformen in der Literatur durchzusetzen. Daß diese Sprache den Status einer $>$ Nationalsprache haben soll, daß Sprache und Literatur dazu beitragen, nationale Identität auszubilden, und dennoch auf Er- 
fahrungen und Erwartungen >des Menschengeschlechts $<$ bezogen werden, gehört zu den Antinomien des Klassik-Programms. Es ist darin den widersprüchlichen Tendenzen seiner Entstehungszeit verbunden.

Die Spannungen zwischen harmonisierend-utopischem Entwurf und aktueller Realität sind ebenso wie die zwischen ästhetischem Postulat und einzelnem Werk den Autoren und ihren Kritikern um 1800 durchaus gegenwärtig. Gerade weil durch die spezifischen Denk- und Anschauungsformen im kulturellen Erfahrungsbereich die politisch-soziale Wirklichkeit der Zeit überschritten und überwunden, durch Form und Stil distanziert oder >geläutert ‘ werden soll, verstehen die >Klassiker ihr Kunstprogramm nicht als ein überzeitlichgültiges. Dem ralten Goether erscheint seine Weimarer Klassik als eine Lebensstation, als historisches Ereignis. In der bildungsbürgerlichen Aneignung der Kunstprinzipien der Klassik [11] wird jedoch der universale Anspruch, aus der Erfahrung des schönen Scheins der Kunst das Leben gestalten, den Menschen und die Gesellschaft verändern zu können, für `klassische Kunstく absolut gesetzt und normativ gewendet [12]. Damit sind Widersprüche, die sich aus den zeitgeschichtlichen Bedingungen und Funktionen des Kunstanspruchs der Weimarer Klassik ergeben, stillgelegt und abgebaut. Sie verweisen auf >Glanz und Elend، der Kunstpraxis im sbürgerlichen Zeitalter،. In verschiedenen Phasen, in denen die Weimarer Klassik neue Aktualität erhält, werden sie wieder erkannt und in das Klassik-Bild eingebracht. Eine solche kritische Aneignung des Klassik-Paradigmas verhindert dann auch, daß mit dem normativen Verständnis >des Klassischen ^ literarische Entwicklungen blockiert werden, die sich gegen die Kunstprinzipien der Weimarer Klassik wenden.

Spannungen im Kulturprogramm der Klassiker sind aber nicht nur in den programmatischen Konstellationen zu sehen - etwa daß durch Rückwendung zur Vergangenheit dem >Menschengeschlecht`seine eigentliche Zukunft eröffnet werden könne oder daß allein eine sautonomer und szweckfreie Kunst die Zwecke menschlicher Bildung und gesellschaftlicher Veränderung zu verwirklichen vermöge. Entschiedene Hinwendungen zu modernen Entwicklungen sind festzustellen, wenn man verfolgt, wie >die Klassiker ( ihre Vorstellungen im >literarischen Leben` um 1800 durchsetzen. Vor allem Goethe nutzt den materiellen Spielraum und das soziale Prestige des neuen Dichteramtes - Möglichkeiten also, die renommierten Autoren aus dem Selbstverständnis des freien Schriftstellers erwachsen. Dieses Rollenbild zeigt sich in der neuen Souveränität des Autors gegenüber Verlegern, Kritikern und Publikum, denen man doch zugleich entgegenkommen muß, um im Gespräch und im `Geschäft‘ zu bleiben. Der literarische Markt wird sowohl als Ort der Selbstprofilierung und Verwirklichung individueller Ansprüche wie als Fessel erfahren. Goethe und Schiller stellen sich dem Prinzip der Ideenkonkurrenz, die aber auch den Kampf um Honorare, um Prestige und Einfluß einschließt, die Innovation als Herausforderung zu höheren Kunstleistungen begreift und zugleich pervertiert sieht im Diktat der literarischen Moden. Im kundigen und geschäftstüchtigen Umgang mit den Medien einer neuen kulturellen Öffentlichkeit - den Werkausgaben, 
Zeitschriften, Almanachen, Jahrbüchern usf. - erweist sich, daß die Klassiker ihr Kulturkonzept zwar dem \Zeitgeist` entgegenstellen, es jedoch mit zeitgemäßen Mitteln zu verwirklichen suchen.

In der Klassik-Rezeption des 19. Jahrhunderts, im Betonen der zeitlosen Kunstleistungen der Weimarer Klassik unter dem normativen Anspruch des Klassischen, ist diese Einbindung des Kunstprogramms in eine - durchaus problematische - Kunstpraxis der modernen Gesellschaft verdeckt und verdrängt. Damit gehen auch die konvergierenden Verbindungen und die kontroversen Bezüge zu anderen literarischen Konstellationen und Programmen im Ausgang des 18. und zu Beginn des 19. Jahrhunderts verloren. Sowohl in der Kritik an der Weimarer Klassik (die intensiv nach 1820 einsetzt) wie in der KlassikVerehrung wird die historische Konstellation aufgebrochen und in einzelne Elemente zerschlagen - zum Zweck der Aggression oder der Legendenbildung. Erst nach 1850 entwickelt sich - besonders im Zeichen der Schiller-Feiern von 1859 - ein relativ homogenes und spannungsloses Bild von der Weimarer Klassik als nationalem Bildungsbesitz und als Verpflichtung zu einer Kunstblüte, deren Erneuerung nur über die politische und soziale Einheit der deutschen $\mathrm{Na}-$ tion zu erreichen ist. Mit der Reichsgründung wird die Bedeutung der Klassik als Grundlage >moderner Geistesbildung` und als kulturelle Garantie für Einheit und Größe der Nation gefestigt. In der Rezeption Schillers und Goethes erhält die Weimarer Klassik den Status eines Dogmas, eines unzerstörbaren Monuments deutscher Kulturleistung.

Gegen die Erstarrung der `bürgerlichen Kulturpraxis` in nationaler Traditionsbindung und selbstgefälligem Bildungsprestige ist der kulturelle Umbruch gewendet, der um 1890 mit dem entschiedenen Bekenntnis zu den ungelösten Problemen von Gegenwart und Zukunft erfahren und reflektiert wird. Er reicht jedoch nicht weit genug, um das privilegierte Paradigma der Weimarer Klassik - über die Auseinandersetzungen im Bereich der Elite-Kultur hinaus - nachhaltig zu erschüttern. Zwar entwickeln sich aus dem heftigen Pro und Contra zu Programm, Aneignungsgeschichte und Aktualität der Weimarer Klassik neue Konstellationen. Doch selbst in den Diskussionen der kulturellen Elite stehen neben dem entschiedenen Traditionsbruch - "Klassik als Muster ohne Wert «und dem Bezug auf 'gegenklassische (Autoren die Rechtfertigungen im Zeichen der Klassik. Die Positionen von Weimar werden erneuert oder fortgeschrieben (vgl. den Naturbegriff, die Lebenskultur, die Rolle von Anthropologie und Mythologie, die Betonung des Autonom-Ästhetischen sowie der sinnstiftenden und disparates Wissen integrierenden Leistungen der Literatur). In Werk und Biographie der beiden Klassiker entdeckt man >moderne Aspekter. Vor allem Goethes Lebensgeschichte erhält die Dimension eines Mythos: ,Überlebensgroßc erscheinen die Fülle der Lebenserfahrungen und die Kraft, ein solches Leben zu meistern und in Kunst zu verwandeln. Auch die Identifikation der 1919 in Weimar gegründeten Republik als >Weimarer Republik ‘ verweist auf die anhaltend geschichtsmächtige und gesellschaftlich institutionalisierte Rolle der Weimarer Klassik. 
In einem strukturellen Vergleich des Kulturwandels zur Zeit der Weimarer Klassik und um 1900 erscheint der programmatische Aufbruch in die Moderne des 20. Jahrhunderts als Wiederholung der Situation am Ende des 18. Jahrhunderts - gleichsam als zweiter Schritt in die Moderne, bei dem nun die Weimarer Klassik als Bezugspunkt gegensätzlicher Kunstauffassungen die Funktionen übernimmt, die gut einhundert Jahre zuvor der Antike zugedacht wurden. Doch läßt sich eine solche funktionale Analogie nur auf der Ebene weitgehender Abstraktion herstellen. Um 1900 ist , Weimar ' ganz anders präsent als >Griechenland $₫$ in den siebziger und achtziger Jahren des 18. Jahrhunderts. Und entscheidend: es sind weiter die Gesetze und Widersprüche der >bürgerlichen Kunstpraxis`, identischer Bedürfnisse und Erwartungen, in denen das späte 18. und das ausgehende 19. Jahrhundert verbunden sind [13]. Um 1900 - so wäre als Annahme zu formulieren - vertiefen sich die Aporien in der Zuordnung von Kunst und Gesellschaft, beschleunigt sich das Tempo der Innovationen und Wandlungsprozesse, verschärft sich die Konkurrenz der Autoren, Verlage und Programme. Daß die Weimarer Klassik dabei in höchst unterschiedlicher Weise interpretiert und `benutzt ` werden konnte, verweist auf die inhärenten Spannungen im Kunstanspruch der Klassiker und auf den Mangel an geschichtlichem Verständnis in den aktualisierenden Bezugnahmen. Außerhalb der ideologisch abgesicherten Herrschaft des Paradigmas besteht jedoch eine zusätzliche Dimension, die auch die jüngste Wirkungsgeschichte der Weimarer Klassik prägt und den eilfertigen politischen Gebrauch der Klassiker erschüttern kann. Entwürfe und Widersprüche des Kulturprogramms von Weimar erscheinen als ein Problempotential, das durch die gesellschaftlichen Entwicklungen bis heute nicht bewältigt oder aufgehoben ist. Es geht um die denkbaren und in der Kunst darstellbaren Ideen von Humanität der Gesellschaft und Selbstverwirklichung des Individuums, die in der geschichtlichen Realität erst noch einzulösen sind.

In den divergierenden gesellschaftlichen Prozessen der deutschen Geschichte nach dem Zweiten Weltkrieg wird nun die Möglichkeit, sich auf Anspruch und Erbe der Weimarer Klassik berufen zu können, zum wiederkehrenden politischen Faktum. Dabei hat das - wirkungsgeschichtlich eingespielte-Gegeneinander von Klassik-Kritik und legitimierendem Klassik-Bezug auch in den letzten Jahrzehnten sein Übergewicht in der Klassik-Verehrung, die unterschiedlich begründet ist. Nach 1945, nach der Barbarei des Hitler-Regimes, werden die normative Funktion der beiden Klassiker (vor allem in Goethes Rolle) und ihre 'zeitlose Modernität ‘ in Ost und West mit konkurrierenden, mitunter auch konvergierenden Interpretationen und Aneignungen bestätigt. Setzt man mit dem Ende der sechziger Jahre, mit der politischen Anerkennung zweier deutscher Staaten und der `Kulturkrise in der Bundesrepublik eine erneute Zäsur im kulturellen Prozeß [14], dann erscheint die Weimarer Klassik abermals als ein Bezugspunkt zur Abgrenzung und Selbstvergewisserung in einer veränderten Konstellation der Moderne. Die Frage, ob die kulturelle Orientierungskrise in der Bundesrepublik eine bewußtseinsgeschichtliche Schwelle zu einer $>$ nachbürgerlichen Kultur der Postmoderne ‘ darstellt und ob in der DDR eine spezifi- 
sche Kultur der entwickelten sozialistischen Gesellschaft geschaffen wird, muß zunächst ohne Antwort bleiben. Zur Debatte stehen Prozesse, die heute noch nicht abgeschlossen und in ihrer Bedeutung einzuschätzen sind. Nur so viel ist festzuhalten, daß seit Beginn der siebziger Jahre das Paradigma der Weimarer Klassik im kulturellen Leben und in der literaturwissenschaftlichen Diskussion in der Bundesrepublik in rascher Folge unterschiedliche Wertungen erhalten hat.

3.

In diesem Zeitraum geht die Literaturwissenschaft weithin davon aus, daß gegenüber dem wirkungsgeschichtlich errichteten Anspruch, in der Literatur der Weimarer Klassik `Musterhaftes`, vorbildliche Leistungen und ein überzeitlich gültiges Kunstverständnis zu finden, Intention und Bedeutung des kulturellen Paradigmas historisch zu analysieren und zu relativieren sind. Die ideologiekritische $>$ Klassikerschelte ‘ der frühen siebziger Jahre [15] erwächst vornehmlich aus dem Protest gegen den normativen Gebrauch des Klassik-Begriffs. Sie arbeitete vielfach mit polemischen Konstruktionen, die vor allem auf die Position Goethes als >Dichterfürst $<$ zielen. Heute ist dieser Kritik am politischen Versagen der Klassiker eine genauere funktionsgeschichtliche Betrachtungsweise gefolgt [16]. Sie beschränkt sich freilich auf die Institutionalisierung des autonomie-ästhetischen Prinzips in der ১bürgerlichen Kunst`, woraus sich wiederum die entschiedene Abgrenzung von hoher und trivialer Literatur herleitet [17]. Die gegenwärtige Diskussion zur Geschichte der idealistischen Ästhetik verspricht Ergebnisse[18], die zum einen die inneren Widersprüche des Autonomieprinzips im gesellschaftlichen Assoziationsbereich seiner zentralen Begriffe (z. B. Freiheit und Herrschaft) aufzeigen, zum anderen aber auch die reduktivharmonisierende Aneignung des Autonomiegedankens im 19. und 20. Jahrhundert nachweisen können.

Bereits Anfang der siebziger Jahre wurden in der Fachdidaktik (im Zusammenhang der Kanon- und Lehrplandiskussionen) die fachwissenschaftlichen Kontroversen zur Weimarer Klassik vielfach verschärft - angesichts des Problems, das historisch Fremde in vertretbarem Rahmen zu aktualisieren. Dazu kamen unmittelbare Einwirkungen kulturpolitischer Vorstellungen. Die Revisionen des >alten Kanons ‘ [19], die Lesebuchdiskussionen und die curriculare Planung von Unterrichtsreihen (vgl. die Literaturangaben in den Beiträgen von I. Ackermann und W. Gebhard) rechtfertigen und profilieren sich bis heute im Umgang mit den Klassikern von Weimar. Sie scheitern aber dort, wo sie sich auf den Bildersturm beschränken. Aus den didaktischen Diskussionen sind Tendenzen hervorgegangen, die in der Mitte der siebziger Jahre dominierten und die Positionen des Pro und Contra zur Klassik im subjektivierenden Zugang auflösten: im Bekenntnis zum wechselnden `Gebrauchswert ‘ der Klassiker für die Selbstfindung und Selbstdarstellung des Ichs im Medium Literatur [20]. Diesem Trend sind auch die gleichzeitigen spektakulären Klassiker-Inszenie- 
rungen zuzuordnen, in denen die Texte Regisseuren, Dramaturgen und Schauspielern zum 'frei interpretierenden Gebrauch ‘ überstellt wurden.

Mit dem Beginn der achtziger Jahre zeichnen sich zugleich eine normative Restitution und eine Re-Historisierung der Klassik ab, die von einem unterschiedlichen Verständnis des Klassik-Begriffs ausgehen. Die Verlage nutzen die kulturelle Betriebsamkeit des Goethe-Jahres zu Klassiker-Editionen, die über Goethe und Schiller hinaus den Hort der rzeitlos-wertbeständigen $<$ Literatur mit der Autorenreihe von Klopstock bis Nietzsche füllen (vgl. die sechzigbändige Bibliothek deutscher Klassiker). Politiker rufen dazu auf, sich wieder der - vermeintlich eindeutigen - Werte klassischer Literatur, des 'Guten, Wahren und Schönen<, zu besinnen.

In der literaturwissenschaftlichen Diskussion scheint sich dagegen die Tendenz durchzusetzen, den Klassik-Begriff auf das historische Ereignis der Weimarer Klassik zu beschränken oder ganz aufzugeben. 1972 versuchte Heinz Otto Burgers Wege der Forschung-Band noch die verschiedenen Perspektiven von Klassik (die `klassische Antike`, die Beispielautoren des Grammatik-, Stilund Rhetorikunterrichts, die nationalen >Blütezeiten ‘ der Kunst, die hochrangigen Kunstleistungen) abzugrenzen[21], während 1974 Manfred Windfuhr ungestüm »den Klassikbegriff zu erledigen « hoffte[22]. Vier Jahre später reagierte Friedrich Sengle gelassen: "Verzichten wir ruhig auf das Wort Klassik. [...] Sprechen wir ganz schlicht von einer bedeutenden Kultur [nämlich der von Weimar] [ [23]. Der Anspruch des `Musterhaften` wäre dann als Resultat einer privilegierten Wirkungsgeschichte zu sehen, die - in kritisch-hermeneutischer Reflexion - mit den Wirkungsgeschichten der sneben- und gegenklassischen Positionen und Programme zu vergleichen ist. Dazu können im literaturgeschichtlichen Verständnis die Voraussetzungen geschaffen werden. Nicht kleinteilige Perioden sind zu bilden, die sich an 'Bewegungen ` wie Sturm und Drang, Klassik, Romantik orientieren, sondern größere zeitliche Zusammenhänge als `Epocher zu entwerfen - also etwa der Zeitraum 1770-1830. Dabei treten die Gleichzeitigkeit des Ungleichzeitigen, kontinuierliche 'Entwicklun-

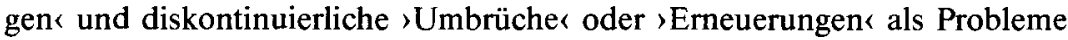
der literaturgeschichtlichen Darstellung in den Vordergrund. Der besondere Stellenwert der Weimarer Klassik, der in einem solchen historischen Umfeld zu diskutieren wäre [24], läßt sich durch die Erweiterung der literaturgeschichtlichen zu einer kulturgeschichtlichen Perspektive besser begründen. Sie kann zum einen dazu beitragen, Selbstverständnis und Intentionen der Autoren in einem größeren Kontext zu erschließen, zum anderen für die Rezeptions- und Wirkungsgeschichte den notwendigen Bezugsrahmen bilden [25]. Der Kulturbegriff, der dabei zu verwenden ist, muß die Wechselwirkung des kulturellen Geschehens mit der gesellschaftlichen Realität verfolgen - beispielsweise in der sozialen Funktion kulturell vermittelter Normen, Einstellungen und Deutungsmuster, die in den wissenschaftlichen Diskussionen ebenso aufzusuchen sind wie in den literarischen Texten und den Ausprägungen des Alltagsbewußtseins. Im historischen Rückblick entstehen Kunstanspruch und Kunstleistung der 
Weimarer Klassik eben auch aus der besonderen Stellung des Kunstprogramms zu den Traditionen und zeitgenössischen Konstellationen der geschichts- und rechtsphilosophischen, der naturwissenschaftlichen und anthropologischen Erfahrungsbereiche[26]. Diese umfassende Perspektive, mit der die Kategorie >kulturelles Paradigmar gerechtfertigt werden kann, setzt voraus, auch nicht-literarische Texte zu berücksichtigen und auf interdisziplinäre Fragen einzugehen[27] - sowohl in der Darstellung des zeitgeschichtlichen Rahmens der Weimarer Klassik als auch in der Reflexion der Wirkungsgeschichte. Beispielsweise wären dabei die Institutionen, Normen und Rollen für die Vermittlung des kulturellen Paradigmas und seiner gesellschaftlichen Funktionen darzustellen. In diesem Band kann eine solche erweiterte Diskussion nur in ersten Ansätzen entwickelt und durchgeführt werden.

Bereits in die Entstehung des Kulturprogramms der Weimarer Klassik sind übergreifende raum-zeitliche Zusammenhänge von Anfang an einbezogen. Es begründet und profiliert sich in der Aneignung und Erneuerung bestimmter Traditionen und durch Abgrenzung von zeitgenössischen Erfahrungen, Positionen und Bewegungen. >Klassik ‘ als Weimarer Klassik zu verstehen, schließt zugleich ein, daß die Rolle des Weimarer Hofes und die Positionen von Goethe und Schiller gegenüber dem Fürstenhaus zu beschreiben wären. Das Erscheinungsbild der Weimarer Klassik wird zudem geprägt von den literarisch-publizistischen Aktivitäten anderer Autoren von Weimar, die sich der zentralen Rolle von Goethe und Schiller unterordnen [28]. Mit den neuen Möglichkeiten des Buchmarktes und des Zeitschriftenwesens wirkt $>$ Weimar ( jedoch über den mitteldeutschen Raum hinaus und kann so zum Kristallisationspunkt einer > Nationalkultur erhoben werden.

4.

Auch dort, wo >Klassikı als eine literaturgeschichtliche Epoche bezeichnet wird, zeigen sich vielfach normative Verengungen. Der Zeitraum wird mit unterschiedlichen Daten abgegrenzt, je nachdem, ob man die klassische Höhe der Kunstleistungen bereits bei Klopstock und Lessing erreicht oder erst mit Goethes Italien-Reise begründet sieht; ob Schillers Tod, Goethes Selbstvergewisserung für den Weg nach Weimar in Dichtung und Wahrheit, die politischen Ereignisse der Jahre 1814/15 oder Goethes Tod das Ende der Klassik in Deutschland bedeuten sollen. Gänzlich unbrauchbar als Epochenbegriff ist 'Klassik ‘, wenn eine weitere Epoche `Romantik ‘ angesetzt wird [29], die dann in einem mehr oder weniger großen Zeitraum - synchron zu Klassik stünde. ,Weimarer Klassik « und >Romantik s sind nicht nur in einem zeitlichen Nebeneinander verbunden. Goethe hat gegenüber Herzog Karl August die Entwicklung der Universität Jena im ausgehenden 18. und frühen 19. Jahrhundert zu einem Zentrum sfortschrittlicher Wissenschaft vertreten und weitgehend geschützt; er hat sich in dieser Einstellung auch nicht durch 'gegenklassischer Po- 
sitionen, die sich in Jena organisierten, beirren lassen. Allein schon damit ist auf Zusammenhänge zwischen dem vermeintlichen Konservatismus der Klassiker und der Progressivität politischer Einstellungen verwiesen, die Jenaer Gelehrten zugeschrieben wurden, und auch auf eine Nähe von klassischem und romantischem Kunstprogramm, die nicht nur als räumliche Nachbarschaft der Gesprächskreise von Weimar und Jena zu verstehen ist. So wäre als annäherungsweiser Epochenbegriff allenfalls >klassisch-romantisch (oder 'Kunstperiode ( ) tauglich. Dabei sind jedoch die verschiedenen Entwürfe der Autonomie-Ästhetik und des symbolisch-allegorischen Kunstbegriffs als Charakteristika der Epoche ausgewiesen und die gleichzeitigen Positionen im Zeichen eines pragmatisch-gesellschaftlichen Verständnisses der Kunstfunktion vernachlässigt.

Wenn in unserem Band für den Zeitraum von 1770 bis 1830 der Begriff >Goethezeit`verwendet wird, so ist er hilfsweise eingesetzt. Er soll nicht im Sinne der Perspektiven und Wertungen gebraucht werden, die Hermann A. Korff im Geist der Goethezeit in die Literaturgeschichte eingebracht hat. Mit >Goethezeit` sei die Notwendigkeit signalisiert, bei literaturgeschichtlichen Gliederungen zunächst von größeren Perioden auszugehen. Sie erlauben es, prägende Prozesse und Strukturen als übergreifende Zusammenhänge zu erkennen. Darin würden dann markante Ereignisse, literarische Programme und kulturelle Bewegungen zu `kleinräumigen ` Abgrenzungen benutzt werden können, ohne sie im Sinne von epochaler Kontinuität und Konvergenz aufeinander beziehen zu müssen [30]. Die Konstruktion einer >Großepoche ‘ von 1770 bis 1830 erfaßt den historischen Vorgang, daß eine nationale Kulturpraxis als Programm entwickelt wird und an Realität gewinnt. Sie erhebt im Gegensatz zu den Partikularkulturen der Höfe, Klöster, Städte, Akademien und Universitäten der vorausgehenden Jahrhunderte einen >universalen` Anspruch. Freilich kann das angestrebte kulturelle Niveau nur gehalten werden, indem - im Widerspruch zum universalistischen Gestus - im Bereich der Literatur die 〉Kunst‘ von Dilettantismus und Lohnschriftstellerei, das 'Schöne und Wahre vom Tendenziösen, Trivialen und bloß Unterhaltenden unterschieden werden. Für die damit notwendigen Selbstbestimmungen und Abgrenzungen der hochzuwertenden >Poesie liefert das Kunstprogramm der Weimarer Klassik entscheidende Argumente.

Die Grenzen, die dabei zu ziehen sind, werden in dieser Konstitutionsphase einer >modernen Kultur immer wieder neu festgelegt. Der Bereich von >Literatur als Kunst، ist theoretisch vor allem durch die philosophische Ästhetik bestimmt und in den Poetiken des 19. Jahrhunderts beschrieben. Doch entsteht in der Kunstpraxis der Goethezeit durchaus ein heterogenes Bild - durch die Lust an Innovation und Experiment und durch den Zwang, literarische Positionen in der Konkurrenz der Meinungen und des Marktes unter Herausforderung geltender Prinzipien zu profilieren. Diese Heterogenität ist in der Literaturgeschichte wiederholt auf einfache Gegensätze reduziert worden - wie etwa klassisch und romantisch, politisch und unpolitisch, konservativ und fortschrittlich. 
Dabei sind vielfach aus den zeitgenössischen Diskussionen, die es historisch zu verstehen und zu analysieren gilt, nur diejenigen Oppositionen übernommen worden, die von Kontrahenten zu strategischen Zwecken der Selbstdefinition und Abgrenzung gebraucht wurden. Weiterführende Untersuchungen müßten - auf einer abstrahierenden Ebene - die ' Tiefenstrukturen < dieser Konstellationen als >Problempotential« der Epoche ermitteln und beschreiben.

Beließe man es für die `Großepoche` 1770-1830 bei dem Begriff `Goethezeit ‘, entstünde die Gefahr, daß die Figur Goethes und - in ihm repräsentiert die klassische Kultur von Weimar zur selbstverständlichen Mitte und zum zentralen Bezugspunkt für das literarische Geschehen in diesem Zeitraum erhoben würden. Eine solche Festlegung könnte nur als Endergebnis einer wertenden Akzentsetzung in der umfassenden literarhistorischen Rekonstruktion akzeptiert werden, sie darf aber nicht die historischen Gegebenheiten verstellen. Erst nach 1808 (dem Jahr des Zusammentreffens von Napoleon und Goethe) rückt der >Alte von Weimars in den Mittelpunkt der Öffentlichkeit, werden literarische Positionen immer wieder mit der Nähe und Ferne zu Goethe bestimmt, wird die Mißachtung durch Goethe traumatisch erlebt (wie etwa bei Kleist). Die Kritik am `Geheimen Rat ` im herzoglichen Dienst, am `Olympier ‘, der mediokre Talente fördert und große Begabungen nicht erkennt, verhindert nicht, daß Weimar bis in die zwanziger Jahre des 19. Jahrhunderts zum Mekka derjenigen wird, die Kunst und >Bildung ‘ als Bastion in den Wirren der Zeit ansehen [31] oder in Weimar den Grundstein zu einer Karriere in der Gunst Goethes legen wollen. Dennoch sind >Weimar< und Goethe nicht der archimedische Punkt kultureller Entwicklung, wie der Begriff `Goethezeit` nahelegen könnte. Solche vorsorglichen Einwände sind notwendig, weil man diese Epochen-Klassifikation in den letzten Jahren wieder häufig verwendet. So wird etwa in der angelsächsischen Germanistik 'Goethezeit ‘ vergleichsweise unbefangen gebraucht. Die Festschriften für Victor Lange (mit dem Titel Aspekte der Goethezeit [32]) und Stuart Atkins [33] oder Terence Reeds Darstellung Die klassische Mitte. Goethe und Weimar 1775-1832[34] beziehen sich zumeist auf den oben bezeichneten Zeitraum, sie begründen ihn jedoch nur durch die >Zeitgenossenschaft $<$ mit Goethe.

In der DDR führen die Literaturwissenschaftler derzeit eine intensive Diskussion, um die Epoche vom Ende des 18. bis in die Anfänge des 19. Jahrhunderts abzugrenzen und inhaltlich zu bestimmen [35]. Ausgangspunkt ist zum einen der Begriff des 'klassischen Erbes` (vgl. in diesem Band S. 562-569), der entweder im Sinne von 'vorbildliche Autoren und Texte der literarischen Tradition oder als Literatur der deutschen Klassik (in einem erweiterten KlassikBegriff von Lessing bis Heine, mit dem Kernbereich Goethe/Schiller) interpretiert wurde. Auch in diesen Debatten zeigt sich eine deutliche Abkehr vom normativen Verständnis ( 'vorbildliche Kunstleistung () und eine Hinwendung zu einem historischen Verstehen. Zudem werden neue Antworten für die Frage gesucht, inwiefern Autoren der bürgerlichen Literatur `mustergültig ‘ für die entwickelte sozialistische Gesellschaft sein können. Weiterhin ist die historische 
Relevanz von $>$ Klassik $₫$ zu überdenken: sollen nur einzelne - wiederum mustergültige - Autoren aus dem Zeitraum der sich konstituierenden `bürgerlichen` Literatur eingeschlossen sein oder gilt `Klassik ‘ür eine bestimmte Epoche? Seit den Jubiläen und Gedenkjahren für Hölderlin und Kleist und seit der Neubewertung der Romantik (die sich Mitte der siebziger Jahre abzeichnete) verstärkt sich die Tendenz, Klassik und Romantik als geschichtliche Einheit zu sehen [36]. Darüber hinaus ist die Periodisierung 1789-1830 mit ihren Abgrenzungen durch politische Ereignisse zumindest für das frühere Datum in Frage gestellt. 1750 (um Lessing und Klopstock einzubeziehen), 1770 (mit Blick auf den sSturm und Drang und den Beginn des dichterischen Werkes von Goethe) oder die achtziger Jahre (mit der Entwicklung einer publizistisch-literarischen Öffentlichkeit [37]) werden als Grenzdaten diskutiert, so daß sich weithin Übereinstimmung mit der Periodisierung im Zeichen des Goethezeit-Begriffes ergibt. Die Beiträge der Reihe Impulse [38] bewegen sich in diesem Zeitraum und erfassen neben den literarischen Texten im engeren Sinne auch die literaturbezogenen Diskurse, das publizistische, populärwissenschaftliche und wissenschaftliche Schrifttum. So entsteht ein differenziertes und kulturgeschichtlich orientiertes Epochenbild, in dem lediglich die heute mindergewerteten Autoren, die Unterhaltungs- und Trivialliteratur, zu kurz kommen.

Für die aktuellen Diskussionen und Forschungsprojekte in der DDR und in der Bundesrepublik zum Komplex der `Goethezeit` wird es ein wichtiges Ziel sein, für diese Phase eines historischen Umbruchs die Leistungen der Weimarer Klassik und zugleich die Grenzen der sie tragenden Personen, Positionen und Perspektiven zu bestimmen und mit den aktuellen Erfahrungen einer erneuten Zäsur in der Kultur- und Gesellschaftsgeschichte zu verbinden.

5.

Der vorliegende Band möchte dazu beitragen, die Geschichtlichkeit der Weimarer Klassik und ihrer Wirkung bewußt zu machen und ihre Funktion im kulturgeschichtlichen Prozeß zu analysieren. Die hier vereinten Beiträge sind in sich geschlossen und können auch unabhängig voneinander bestehen. Aber im gemeinsamen thematischen Argumentationsrahmen, den direkten wie den mittelbaren Beziehungen zueinander, dem Arrangement zu einem Bild geschichtlicher Konstellationen und Prozesse verstehen sie sich gleichzeitig als Teile eines übergeordneten Ganzen.

Der erste Teil des Bandes faßt Beiträge zusammen, denen es in besonderer Weise darum zu tun ist, den Klassik-Begriff weiter zu profilieren und z. T. auch zu problematisieren. Dabei überwiegt das Engagement für Perspektiven, die bislang noch kaum weiter verfolgt und ausgeleuchtet wurden. Daß die Begegnung mit der Antike, vor allem der griechischen Klassik, zu den Voraussetzungen der Weimarer Klassik gehört, ist uns geläufig. Weniger bekannt ist, wie sich an dem Problem der Aneignung - unter bedeutsamer Vermittlung der Altphilo- 
logen - eine eigene, von der Aufklärung deutlich verschiedene Hermeneutik der Weimarer Klassik herausbildet, mit der ausgeprägte kulturpolitische Zielsetzungen verbunden werden (Manfred Beetz). Das Verhältnis von Weimarer Klassik und Französischer Revolution ist in jüngster Vergangenheit mehrfach diskutiert worden. Die Beziehungen zwischen Schillers Briefen Über die ästhetische Erziehung des Menschen und der konservativen Revolutionskritik Edmund Burkes blieben bis heute unberücksichtigt. Ihr Nachweis belegt in der Differenzierung >rhetorischer` und 'ästhetischer( Revolutionskritik zugleich grundsätzliche Unterschiede von konservativer(Theorie wie Praxis der Revolution verwerfender) und liberaler (das theoretische Fundament der Revolution bejahender) Revolutionskritik (Dieter Borchmeyer). Die Ausbildung der modernen Gesellschaft seit etwa 1770 führt auch zu einer neuen Festlegung der Geschlechterrollen. Die in der fiktionalen wie expositorischen Literatur zwischen 1770 und 1805 lebhaft geführte Diskussion darüber dokumentiert ein breites Spektrum von Auffassungen, das von Zeichen einer konservativen Männerfronde bis hin zu ausgeprägten progressiven und emanzipatorischen Tendenzen reicht, die freilich eher Ausnahme bleiben (Volker Hoffmann). Andere Beiträge nehmen die Enge des Begriffs der , Weimarer Klassik ‘ als Problem auf. Bereits im zeitgeschichtlichen Kontext betrachtet, impliziert er eine fragwürdige Selektion, die an der auffälligen Umwertung und Klassik-Ausbürgerung Wielands gezeigt wird, der dabei in besonderem Maße als frühes Opfer einer nationalliterarischen Konzeption der Literaturgeschichte gesehen wird (Herbert Jaumann). Auf andere Weise wird die Enge des Klassik-Begriffs fragwürdig, wo allgemeine Probleme unserer Epochenbegriffe zur Diskussion stehen (Michael Titzmann): Nicht als Epoche, sondern nur als sstruktureller Typus $<$, als >Richtung innerhalb einer weiter gefaßten epochalen Einheit von 1770 bis 1830 - nenne man sie /Goethezeit‘ oder anders - läßt sich die Weimarer Klassik danach verstehen.

Die Beziehung der Weimarer Klassik zum sozialen Wandel seit 1770, das Postulat eines großräumigeren Epochenbegriffs, das Bewußtsein einer Privilegierung der Weimarer Klassik im kulturgeschichtlichen Prozeß: das alles legte Erweiterungen der geschichtlichen Perspektive nahe. So stellt der zweite Teil des Bandes die Weimarer Klassik in den Beobachtungszusammenhang der Zeit von 1770 bis 1830 . Im Nebeneinander wie in den Überschneidungen konkurrierender Richtungen und Tendenzen werden übergeordnete geschichtliche Determinanten und Prozesse greifbar, wird der Epoche zugleich etwas von jener Vielgestaltigkeit zurückgegeben, die sie durch kleinräumige Parzellierungen und auf dem Wege wirkungsgeschichtlicher Verengungen teilweise eingebüßt hat. In deutlicher Opposition zu allen Enthistorisierungen des Klassikbegriffs werden Fragen der Geschichtlichkeit wieder aufgenommen, die sich bereits im ersten Teil des Bandes ankündigten: Fragen der geschichtlichen Voraussetzungen wie der literarischen Verarbeitung geschichtlicher Erfahrungen. Gegen das Mißverständnis des Weimarer Programms im Sinne eines überzeitlichen Anspruchs macht eine erste Gruppe von Beiträgen deutlich, wie sich Texte der 
Weimarer Klassik gerade als Niederschlag eines intensiven Geschichtsbewußtseins verstehen. Die Mitschuldigen zeigen im Vergleich der verschiedenen Fassungen, die aus der ursprünglichen Farce schließlich das frühklassische Lustspiel machen, eine neue Gewichtung der geschichtlichen Erfahrung (vgl. u.a. die Motive der Zeit, des Geldes und Besitzes, der Erfahrung von Gewalt), der gegenüber die Versöhnung der Welt nur im Bild der Kunst, im Lustspiel gelingt (Wolfgang Stauch-v. Quitzow). An Iphigenie auf Tauris lassen sich verwandte Zusammenhänge beobachten. Die Behauptung des Menschen und seiner Freiheit im Handeln Iphigeniens richtet sich gegen das Bewußtsein gesellschaftlicher Bedingtheit. Die Botschaft der Humanität erscheint motiviert im Zusammenhang einer umfassenden Geschichtsdeutung (Ursula Segebrecht). Die Entstehung der Kraniche des Ibykus erweist sich als charakteristische Etappe der Zusammenarbeit von Goethe und Schiller, aber auch als Ausdruck einer Vermittlung von Kunst, Natur und Gesellschaft, die erst aus der zeit- und publikumsbezogenen Wirkungsabsicht voll verständlich wird (Wulf Segebrecht). Die Stilisierung auf das Idyllische hin, wie sie an der Rezeption des Vicar of $\mathrm{Wa}$ kefield von Oliver Goldsmith zu beobachten ist, gilt es vor dem Hintergrund jüngerer Wirklichkeits- und Zeiterfahrung zu verstehen (Walter Pache). Goethes Rezeptionszeugnisse zwingen uns dabei, die klassische Periode zu überschreiten und das Frühwerk wie das Spätwerk in die Betrachtung einzubeziehen. Erst recht erscheinen die Bezugnahmen auf Cagliostro und den Okkultismus (Klaus H. Kiefer) wie das Erdbeben von Lissabon (Thomas Bourke) als Katalysatoren einer geschichtlichen Standortbestimmung, die das Selbstverständnis der Weimarer Klassik mit betrifft, aber sinnvoll nur im erweiterten epochalen Kontext zu diskutieren ist. Selbst der Ausblick auf Wege der westeuropäischen Literatur wird da mehrfach erforderlich. Wie mit Kleist, so stehen zuletzt auch mit Hölderlin und Achim von Arnim Autoren in Frage, die nicht der Weimarer Klassik zugehören. Die Rückgriffe auf die Antike - bei Hölderlin im Ringen um die Poetik seiner späten Lyrik (Gregor Thurmair), bei Arnim im Einsatz der Mythologie (Gisela Henckmann) - führen denn auch in bezeichnender Weise an der Klassik vorbei. Und doch belegen sie im Rekurs auf die Antike denselben Bezugspunkt: Sie tragen dazu bei, den produktiven Dialog zwischen der eigenen Zeit und der Antike über die Weimarer Klassik hinaus als wesentliche epochale Determinante zu beglaubigen. In allem also Ermunterungen, aus heutiger Sicht die Geschichtlichkeit der Weimarer Klassik zu betonen und ihre Einbindungen in eine vielgestaltige Epoche zu reflektieren. Aber die Betrachtungen im Anschluß an Stielers Goethe-Porträt zeigen auch, daß noch in der 'Goethezeit` eine wirkungsgeschichtlich bedeutsame doppelte Verfremdung der Weimarer Klassik in Gang kommt: in der kultischen Überhöhung zum Bildungsgut von scheinbar geschichtsloser Geltung wie in dem Beitrag zur ästhetischen Verbrämung und Legitimation von Macht (Günter Hess). Im Zeichen solcher - relativ konstanter - kultureller und zugleich politischer Funktionen steht die Wirkungsgeschichte der Weimarer Klassik in der zweiten Hälfte des 19. Jahrhunderts. 
Die folgenden Teile des Bandes bleiben zunächst ganz der privilegierten Stellung der Weimarer Klassik auf der Spur, mit Bezug vor allem auf wesentliche Phasen des 20. Jahrhunderts. An der Zeit zwischen 1890 und 1930 wird eine auffällige Polarisierung der Reaktionen faßbar, in mehreren Beiträgen verdeutlicht durch die Einbeziehung nicht-literarischer Diskurse und interdisziplinärer Fragestellungen. Die Geschichtswissenschaft zwischen Kaiserreich und Drittem Reich zeigt sich bemüht, Bismarck und Goethe weiter einander anzunähern, als es Bismarcks eigenen Intentionen entsprach, um beide als Begründer des modernen Deutschlands zu begreifen (Adalbert Wichert). Die neue Wissenschaft der Psychoanalyse beruft sich auf Goethe, stilisiert ihn geradezu zum "Präfreudianer « (Horst Thomé). Es ist nur die Kehrseite solcher Tendenzen, die Gegenwart in ahistorischer Weise an die Überlieferung anzuschließen, wenn die literarische Moderne des frühen 20. Jahrhunderts - wie am Beispiel des Expressionismus gezeigt wird - sich dann dezidiert am Traditionsbruch definiert und geradezu als das Gegen-Klassische begreift (Michael Stark). Mehrere Beiträge berühren sich darin, daß sie charakteristische Strukturen der Literatur des 20. Jahrhunderts aufgreifen und vergleichend zur Klassik in Beziehung setzen. Das Modell der "Wiedergeburt « zu »neuem Leben « in erzählender Literatur 1890-1930 wird verwandten Vorstellungen der Goethezeit gegenübergestellt, aber mit Entschiedenheit auch als Ausdruck einer epochalen Konstellation des 20. Jahrhunderts gedeutet (Marianne Wünsch). Nachdrücklicher als gegen-klassisches Paradigma erweist sich die literarische Moderne noch einmal in den geänderten Normen der Diskurse über den Tod (Thomas Anz), ebenso in einem veränderten Umgang mit Emotionen, der gleichzeitig dem Leser im Prozeß der Literaturrezeption eine neue Funktion gibt (Karl Richter).

Noch einmal schien die Weimarer Klassik, Goethe vor allem, in der Zeit nach 1945 berufen, bedeutsame politische Funktionen für die Gegenwart zu übernehmen: als Potential programmatischer Menschlichkeit nach einer überstandenen Phase der Barbarei, als Orientierung auf der Suche nach einer neuen nationalen Identität, als geistige Basis gesellschaftlicher Neugründung und materiellen Wiederaufbaus (Maximilian Nutz). An öffentlichen 'Sprechhandlungen der Zeit zwischen 1945 und 1949 wie den Reden bei der Verleihung des Goethepreises, den Festreden anläßlich der Goethefeiern 1949, Vorworten zu Goethe-Anthologien u. a. m. wird das besonders deutlich. Aber wiederum erweist sich, daß sich in der Belebung der Gegenwart aus dem Geist der Vergangenheit auch falsches Bewußtsein etabliert, an dem zwei Jahrzehnte später ein kompromißloser Antagonismus von kulturkonservativer Apologie und schroffer Traditionsabsage aufbricht. Daß die Auseinandersetzung in zentraler Weise eine gesellschaftliche Institution wie die Schule betrifft, wie an der Rezeption der Weimarer Klassik im Schullesebuch (Irmgard Ackermann) und an der Didaktik-Diskussion (Walter Gebhard) gezeigt wird, deutet nur folgerichtig an, $\mathrm{da} \beta$ es in Wahrheit um Grundlagen der Gesellschaft geht. Insofern ist die Frage des Verhältnisses zum 'kulturellen Erbe- - in der DDR wie der Bundesrepublik - auch eine Frage der jeweiligen politischen Kultur. Mehrere Beiträge machen 
sichtbar, wie sich die unterschiedlichen sozialen Gegebenheiten in beiden deutschen Staaten in die unterschiedlichen Rezeptionen der Weimarer Klassik hinein fortsetzen. Aber sie lassen, gerade am Beispiel der literarischen Entwicklung selbst, auch darauf schließen, daß man hier wie dort zu einem reflektierten Traditionsverhältnis unterwegs ist, das sich jenseits von kritikloser Verehrung wie geschichtsblindem Protest als legitimes Element des Gegenwartsbewußtseins begreift. Goethe-)Transkriptionen< im Roman der siebziger Jahre (Ernst Weber) und Vergleiche von Hacks und Handke (Jürgen Sang) zeigen an, wie im vollen Bewußtsein des geschichtlichen Abstands Konvergenzpunkte aufgesucht werden, in denen sich die Folie der Vergangenheit anbot, um Probleme der Gegenwart zur Sprache zu bringen. Im > Dichterroman` der neueren DDRLiteratur verrät sich darüber hinaus eine neue Goethe-Distanz, die das rgelungene Leben` des Geheimen Rates mit dem >unlebbaren Leben` derjenigen konfrontiert, die den Weg nach Weimar nicht gefunden haben: Lenz, Hölderlin und Kleist (Jörg Schönert). Erlangt hier im kritischen Dialog von Gegenwart und Vergangenheit die Erzählprosa ein neues Gewicht, so bleiben Drama und Theater doch bis in die jüngste Zeit hinein an der Reflexion des geschichtlichen Abstands beteiligt. Eine moderne >Dramaturgie der Gewalt', wie sie umschrieben wird, bringt diesen Abstand auch so zur Geltung, daß sie sich in versteckter Weise auf das Scheitern einer klassischen Utopie der gewaltlosen Gesellschaft zurückbezieht (Gunter Reiß). Inszenierungen von Dramen Schillers, der nach 1945 gegenüber Goethe zunächst in den Hintergrund getreten war, liefern Ende der siebziger und Anfang der achtziger Jahre ein interessantes Beispiel, wie sich geschichtliche Rekonstruktion und aktuelle Funktionalisierung verbinden können. Denn die angestrebte Vermittlung von Geist und Körper in der eigenen 'Sinnlichkeit des Theaters ließe sich einerseits auf Funktionen der klassischen Bildungsidee im geschichtlichen Kontext zurückführen; doch angesichts der verschärften aktuellen Auswirkungen von Zweckrationalität, Arbeitsteiligkeit und Mechanisierung gab sie gleichzeitig dem Theater eine neue gegenwartskritische Dimension (Gert Sautermeister).

Auch über die Bindung an das Rahmenthema hinaus haben gemeinsame Interessen wesentlich dazu beigetragen, das übergeordnete Ganze zu profilieren. Wichtig war den Autoren die Erweiterung der Betrachtung von der Literaturzur Kulturgeschichte. Bedeutsam dafür schien immer wieder, nicht-hochgewertete und nicht-fiktionale Texte einzubeziehen, interdisziplinäre Fragestellungen aufzunehmen, auf die Rolle der institutionellen Träger der Klassik-Vermittlung wie Schule und Hochschule, Theater, Bildungsvereine, Feiern und Reden etc. einzugehen. Viele Beiträge verbindet ein ausgeprägtes Interesse an Vermittlungen von Theorie und Geschichte, deren gegenwärtige Literaturwissenschaft in mancher Hinsicht bedarf. Auffällig auch die Aufmerksamkeit für eher verdrängte Dimensionen der Literatur, z. B. das sinnliche und das emotionale Element. Was als Grundhaltung vorwiegt, ist ein kritisch-hermeneutisches Interesse, das gegen den normativen Anspruch des >Klassischen schichtlichkeit wie den Wandel der wirkungs- und rezeptionsgeschichtlichen 
Bedingungen setzt: ein Interesse, in dem sich historische Argumentation und gegenwartsbezogenes Engagement nicht stören, sondern bedingen.

Doch seien, nach einer solchen Betonung von Einheit und Zusammenhang, auch Grenzen eingestanden, die uns deutlich vor Augen stehen. Sie betreffen sowohl die Abfolge der Beiträge als auch die Ausfüllung des Rahmenthemas. Was immer es an behutsamen Abstimmungen gegeben hat: den Anspruch eines systematischen Argumentations- und Darstellungszusammenhangs können und wollen wir nicht erheben. Sinnvolle Gruppierungsmöglichkeiten der Beiträge zeichneten sich zwar bereits während der Vorbereitungs- und Entstehungszeit des Bandes ab. Doch in vielem war die endgültige Reihenfolge erst nachträglich, also nach dem Eingang der Aufsätze, zu entscheiden. Die Weite des Arbeitsgebietes verurteilte zu bewußter Konzentration und Aussparung. Aufgenommen wurden Frageansätze bevorzugt dort, wo es sich um wenig erforschtes Terrain handelte, die Forschungslage deutliche 'Lücken` vorgab. Im wesentlichen übersprungen wurde die Zeit zwischen 1830 und 1890, also weite Teile des 19. Jahrhunderts. Das versteht sich ebenso aus dem Zwang zur Beschränkung wie aus einer Spezifizierung der Fragestellung, der es einerseits um die Geschichtlichkeit und den geschichtlichen Kontext der Weimarer Klassik, zum anderen eben nicht einfach um ihre allgemeine Wirkungs- und Rezeptionsgeschichte zu tun war, sondern um das Spannungsverhältnis von Klassik und Moderne im kulturgeschichtlichen Prozeß. Die weitgehende Aussparung des Nationalsozialismus, auf den immerhin am Rande in einigen Beiträgen Bezug genommen wird, haben wir mit Bedauern registriert. Doch konsequent war sie insoweit, als die `Gleichschaltung` den Anspruch der Moderne unterdrückte und den produktiven Gegensatz von Klassik und Moderne darin aufhob.

6.

Die Publikation, die hier vorgelegt wird, erscheint etwa gleichzeitig mit dem Band Die Geschichtlichkeit der deutschen Klassikvon Walter Müller-Seidel. Ein Kapitel »Weimarer Klassik und Weimarer Republik. Zur Geschichte eines ungeschichtlichen Denkens « wird diesen Band eröffnen. Den Ausführungen zufolge trug das allzu ungeprüfte Fortwirken der Weimarer Klassik, verbunden mit einer Abwehrhaltung gegenüber der literarischen Moderne, wesentlich dazu bei, daß die Zeit der Weimarer Republik ein adäquates Gegenwartsbewußtsein verfehlte. Was die ,Weimar-Ideologie in partiellen Zurücknahmen der Moderne erreichte, erscheint nicht nur als Ausdruck ästhetischer Normenkollisionen, sondern zugleich als Phase im Scheitern der Weimarer Republik. In Auseinandersetzung mit dem gefährlich Politischen eines ungeschichtlichen Denkens plädiert Walter Müller-Seidel für eine Geschichtlichkeit, die das Bewußtsein geschichtlicher Distanz und die Besinnung auf das Eigenrecht der Gegenwart einschließt. Im Nachdenken über die Probleme der Weimarer Repu- 
blik erscheint die Klärung des Traditionsbezugs, zu der auch unser Band beitragen will, unversehens als politische Aufgabe unserer Gegenwart.

Walter Müller-Seidel hat in seiner bisherigen Arbeit die Weimarer Klassik und die Moderne des 20. Jahrhunderts in ihren historischen Voraussetzungen, ihren Erscheinungsformen und ihrem Spannungsverhältnis in vielfältiger Weise erschlossen. An diesen Arbeitsschwerpunkten und am interdisziplinären Interesse des Münchener Literaturwissenschaftlers haben sich die Mitarbeiter dieses Bandes orientiert. Sie haben, so gesehen, sachliche Gründe, ihm diesen Band zum 65. Geburtstag zuzueignen. Aber sie haben auch persönliche Gründe, es zu tun. Als akademischem Lehrer verdanken sie Walter Müller-Seidel entscheidende Erfahrungen und Förderung. Er hat für die meisten Beiträger die Promotion, für viele auch die Habilitation betreut. Sie arbeiten als Assistenten oder Lehrbeauftragte mit ihm zusammen oder haben dies in den letzten zwanzig Jahren getan. Daß sich in solcher Zusammenarbeit ein breites Interessenspektrum und unterschiedliche methodische Vorstellungen entwickeln konnten, zeigt unser Band.

Alle Beiträge erscheinen zum ersten Mal im Druck. Sie sind im Rahmen einer zweijährigen Vorbereitungsphase und vielfach in gegenseitiger Abstimmung entstanden. Die meisten Mitarbeiter hatten im Gegenstandsbereich des Bandes mehrere Vorschläge für ihren Beitrag vorgelegt, aus denen dann die hier versammelten Themenstellungen so ausgewählt wurden, da $ß$ wichtige Aspekte des Leitthemas sowohl in theoretisch-systematischer wie in historisch-interpretierender Sicht angesprochen werden konnten. Entwürfe zu den meisten Aufsätzen wurden im Oktober 1981 bei einem Werkstattgespräch in München gemeinsam diskutiert, ergänzt und aufeinander abgestimmt. Diese Kooperation sollte freilich nicht die Souveränität der Autoren beeinträchtigen oder Gegensätze in Positionen und Argumentationen aufheben. Gemeinsames Ziel war, einen Arbeits- und Gesprächskontakt, der sich über mehrere Generationen von Doktoranden hinweg im Kreis um Walter Müller-Seidel gebildet hat, in einem wissenschaftlichen Projekt zu nutzen. Das Ergebnis ist eine Geste des Dankes an Walter Müller-Seidel. Es ist auch ein Dokument der $>$ Wirkungsgeschichter eines Hochschullehrers, jedenfalls für die Jahre ab 1959, als Walter MüllerSeidel nach München berufen wurde - an jene Universität, der er seither trotz mehreren Rufen treu geblieben ist.

Der Band ist Niederschlag eines unter Hochschulgermanisten geführten Gesprächs, das Übereinstimmungen suchte, aber auch Widerspruch zuließ. Er will sich nicht auf die Abgeschiedenheit akademischer Diskussionen zurückziehen, sondern wendet sich an eine breite Öffentlichkeit - die in Schulen, Theatern und der Kulturpolitik Tätigen vor allem, auch an Vertreter benachbarter Disziplinen.

Die Herausgeber haben in vielfältiger Weise zu danken: dem Verlag, insbesondere Herrn Dr. Bernd Lutz für die außerordentlich gute Zusammenarbeit, der VG-Wort für einen großzügigen Druckkostenzuschuß, den Autoren des Bandes für das kollegiale teamwork،. Das Manuskript wurde im Herbst 1982 
abgeschlossen. Unsere Sekretärinnen Marie-Louise Boden und Gabriele Didier sowie die Mitarbeiter Cornelia Berens, Willibert Kempen und Lutz Tantow haben bei der Vorbereitung der Manuskripte für den Satz, der Satzkorrektur und der Erstellung des Registers geholfen.

Saarbrücken und Aachen, im März 1983

\author{
Karl Richter \\ Jörg Schönert
}

\section{Anmerkungen}

1 Es versteht sich, daß diese Problemskizze lediglich Leitlinien und einzelne Perspektiven für ein solches Unternehmen entwerfen kann. Auf Belegstellen für unsere Ausführungen im schriftstellerischen Werk der angesprochenen Autoren aus Klassik und Moderne wird verzichtet; die neuere Forschungsliteratur ist nur in exemplarischen Positionen und mit sporadischen Verweisen angesprochen.

2 Zum literaturgeschichtlichen Zeitraum der s jüngeren Moderne ( vgl. beispielsweise in jüngster Zeit Benjamin Bennett u. a. (Hg.): Probleme der Moderne. Studien zur deutschen Literatur von Nietzsche bis Brecht. Festschrift für Walter Sokel. Tübingen 1983. - In unserem Band wird die Epoche von etwa 1890 bis 1930 nicht sals Ganzes` behandelt, sondern nur in den wirkungsgeschichtlichen Bezügen zur Weimarer Klassik und Goethezeit. Die Probleme im literaturwissenschaftlichen Umgang mit dem >Moderne<-Begriff sind ausführlicher angesprochen in Theo Elm und Gerd Hemmerich (Hg.): Zur Geschichtlichkeit der Moderne. Der Begriff der literarischen Moderne in Theorie und Deutung. Ulrich Fülleborn zum 60. Geburtstag. München 1982. - Die Herausgeber und die einzelnen Aufsätze verweisen - mit unterschiedlichen Präferenzen - auf die philosophiegeschichtliche Gleichsetzung von Neuzeit und Moderne ( 'kopernikanische Wende (), auf die Einschätzung der Jahrzehnte um 1800 als einer neuen Epoche (im Zeichen der Moderne) im Bewußtsein vieler deutscher Schriftsteller (dazu W. Wittkowski, S. 113-134, und G. Schulz, S. 135-151) sowie auf die Festlegung \Kunst der Moderne als Kunst des 20. und späten 19. Jahrhunderts, die in den Kunstwissenschaften (einschließlich der Literaturgeschichte) überwiegt.

3 Die terminologischen und theoretischen Probleme, die sich mit dem Gebrauch des Kultur-Begriffs und der Argumentation zum Kulturwandel ergeben, können hier nicht diskutiert werden. Zum gegenwärtigen Forschungsstand informiert Otto A. Baumhauer: Kulturwandel. Zur Entwicklung des Paradigmas von der Kultur als Kommunikationssystem. Ein Forschungsbericht. In: DVjs 56 (1982), Sonderheft Kultur. Geschichte und Verstehen, S. 1*-167*. - Wir verwenden $>$ Kultur i im sozialwissenschaftlichen Sinn als spezifischen Aktions- und Erfahrungszusammenhang im System der Gesamtgesellschaft. - Im Zusammenhang der eigentlich notwendigen theoretischen Verständigung über den Kultur-Begriff wäre auch die Verwendung von 'kulturellem Paradigma genauer einzuordnen. Wir beziehen uns dabei auf die soziokulturelle Funktion des Kunstprogramms der Weimarer Klassiker in seiner privilegierten wirkungsgeschichtlichen Rolle.

4 Vgl. die reichhaltige Forschungsliteratur zur Geschichte und Typologie der `Querelle des Anciens et des Modernes`; u. a. Hans R. Jauß: Schlegels und Schillers Replik auf die "Querelle des Anciens et des Modernes«. In H. R. J.: Literaturgeschichte als Provokation (es 418) 2. Aufl. Frankfurt a. M. 1970, S. 67-106, und Peter K. Kapitza: Ein 
bürgerlicher Krieg in der gelehrten Welt. Zur Geschichte der Querelle des Anciens et des Modernes in Deutschland. München 1981.

5 Vgl. dazu Wilhelm Voßkamp (Hg.): Utopieforschung. Interdisziplinäre Studien zur neuzeitlichen Utopie. 3 Bde. Stuttgart 1982.

6 >Entwicklung zur Moderne〈 soll nicht als linearer Proze $\beta$ einer sich ständig steigernden 'Modernisierung in einem Sinne verstanden werden, der dann geschichtsoptimistisch mit dem Fortschrittsgedanken zu verbinden oder pessimistisch im Zeichen der 'Entfremdung ‘, des ' Kulturverfalls‘, der ,Vergesellschaftung der Lebenswelt ‘ usf. zu sehen ist. - Die Kategorie >moderne Gesellschaft « wäre im Anschluß an sozialwissenschaftliche und sozialgeschichtliche Forschungen zu präzisieren; in der Einleitung kam es vor allem darauf an, die eingespielten Wertungen und Zuordnungen im kulturund literarhistorischen Verständnis von >Moderne` im Bezug auf die umfassendere Sicht der Gesellschaftsgeschichte in Frage zu stellen und zunächst eine verfremdende Perspektive zu gewinnen.

7 Vgl. dazu u. a. Deutsche Literatur und Französische Revolution. Sieben Studien von Richard Brinkmann u. a. (KI. Vandenhoeck-Reihe 1395) Göttingen 1974; Harro Segeberg: Deutsche Literatur und Französische Revolution. Zum Verhältnis von Weimarer Klassik, Frühromantik und Spätaufklärung. In Karl O. Conrady (Hg.): Deutsche Literatur zur Zeit der Klassik. Stuttgart 1977, S. 243-266; Lothar Bornscheuer: Deutsche Klassik und französische Revolution. Politisch-soziales Bewußtsein in der klassischen deutschen Literatur und in der Klassik-Forschung. In Julius H. Schoeps u. Imanuel Geiss (Hg.): Revolution und Demokratie in Geschichte und Literatur. Zum 60. Geburtstag von Walter Grab. Duisburg 1979, S. 19-39.

8 Victor Lange: Überlegungen zur »Deutschen Klassik«. In Bernhard Fabian u. Wilhelm Schmidt-Biggemann (Hg.): Das achtzehnte Jahrhundert als Epoche (Studien zum achtzehnten Jahrhundert. Bd. 1) Nendeln 1978, S. 87-103.

$9 \mathrm{Zu}$ den Problemen, die hier im einzelnen nicht erörtert werden können, zählt auch die Kardinalfrage, inwieweit für den geschichtlichen Zeitraum von Ausbildung und Durchsetzung der >modernen Gesellschaft $\$ in der Literaturgeschichte vom $>$ Zeitalter der bürgerlichen Literatur`, der 'Institution bürgerliche Kunstく usf. gesprochen werden kann. - Die neuere Forschung stimmt weitgehend darin überein, daß sich im letzten Drittel des 18. Jahrhunderts ein neues Literaturverständnis und eine entschieden veränderte Konstellation des literarischen Lebens` ausbilden. $\mathrm{Ob}$ man hierfür das Etikett bürgerlich ` benutzen kann, ist bereits strittig. (Vgl. aus der Perspektive der Sozialgeschichte beispielsweise Wolfgang Ruppert: Bürgerlicher Wandel. Studien zur Herausbildung einer nationalen deutschen Kultur im 18. Jahrhundert. Frankfurt a. M. u. New York 1981). Erhebliche Kontroversen ergeben sich, wenn die Endgrenze für diesen Prozeß festzusetzen ist: Soll man sie im Ausgang des 19. Jahrhunderts fixieren, mit dem Ende des Ersten Weltkriegs, mit dem Beginn der Naziherrschaft, mit dem Ende des Zweiten Weltkriegs, mit den späten sechziger Jahren (im Blick auf die Kontinuität `des Bürgerlichen` in der Literatur der Bundesrepublik) oder ist der Prozeß auch heute noch nicht abgeschlossen?

10 Das Denkmuster und Formprinzip von der Transformation der Gegensätze auf eine höhere Bewußtseins- oder Seinsebene ist weiter angelegt und trifft die Kunst-Programmatik der Weimarer Klassik eher als die Formel von der "Klassik als Ausgrenzungsprozeß « - Christa Bürger: Tradition und Subjektivität (stw 326) Frankfurt a. M. 1980, S. 169. Diese Charakteristik beschreibt besser das ästhetische Konzept des >Poetischen Realismus<, das sich auch auf Verankerungen in entsprechenden gesellschaftspolitischen Vorstellungen von der sbürgerlichen Mitte stützt. Von dem Kernbereich der Nation wird all das rausgegrenzt‘, was sich ihm nicht zuordnet. 
11 Vgl. zur Rezeptionsgeschichte Karl R. Mandelkow: Goethe in Deutschland: Rezeptionsgeschichte eines Klassikers. Bd. 1, 1773-1918. München 1980.

12 Diese Normativität ist freilich nicht ausschließlich eine Konstruktion der Wirkungsgeschichte. Sie hat ihre Ansatzpunkte durchaus im Inhaltlichen des Kulturprogramms der Weimarer Klassik und im >Herrschaftsanspruch', mit dem Goethe und Schiller in den neunziger Jahren ihre Vorstellungen durchsetzen wollten.

13 Vgl. Anmerkung 9.

14 Auf die sKulturkrise ( haben vor allem die literaturwissenschaftlichen Arbeitsgruppen um Christa und Peter Bürger in ihren Publikationen verwiesen, zuletzt Peter Bürger (Hg.): Zum Funktionswandel der Literatur (es 1157) Frankfurt a. M. 1983, S. 13. - In der Geschichte der `bürgerlichen Literatur` scheint eine solche krisenhafte Erfahrung ein wiederkehrendes Phänomen zu sein (nach 1830, um 1900, um 1970). Offensichtlich sind die Kriterien, mit denen 'Krise und ,Zusammenbruch können, für den Prozeß des Literatur- und Kulturwandels erst noch genauer zu bestimmen.

15 Vgl. etwa Reinhold Grimm u. Jost Hermand (Hg.): Die Klassik-Legende. Frankfurt a. M. 1971.

16 Zum Beispiel Christa Bürger: Der Ursprung der bürgerlichen Institution Kunst. Literatursoziologische Untersuchungen zum klassischen Goethe. Frankfurt a. M. 1977.

17 Hierzu als neuere Beiträge Christa Bürger u. a. (Hg.): Aufklärung und literarische Öffentlichkeit (es 1040) Frankfurt a. M. 1980 (dort J. Schulte-Sasse, S. 83-115; Ch. Bürger, S. 162-212; O. Frels, S. 213-237) und dies. u. a. (Hg.): Zur Dichotomisierung hoher und niederer Literatur (es 1089) Frankfurt a. M. 1982.

18 Diese wichtige Perspektive zum Verhältnis von Klassik und Moderne tritt - insgesamt gesehen - bei den Beiträgen unseres Bandes in den Hintergrund. Dies muß im Sinne der Re-Historisierung der Debatte kein Schaden sein, denn mit den Konzepten der Ästhetik werden nur die institutionell privilegierten Reflexionen des literarischen Prozesses erfaßt. In einem solchen Status erhalten sie freilich besonderen Einfluß auf den geschichtlichen Wandel, bestimmen und repräsentieren ihn aber nicht ausschließlich. - Zur Forschungsdiskussion, die in den letzten Jahren mit Vehemenz und Erfolg eingesetzt hat, vgl. u. a. Ch. Bürger: Aufklärung (insbesondere R. Grimminger, S. 116132), und Willi Oelmüller (Hg.): Kolloquium Kunst und Philosophie Bd. 1. Ästhetische Erfahrung (UTB 1105) Paderborn 1981. - Bernd Witte (RWTH Aachen) sei herzlich gedankt für hilfreiche Diskussion zum Problem >Autonomie-Ästhetik im Zusammenhang mit seinem Manuskript Genie, Revolution, Totalität. Zum Epochenbewu/3tsein deutscher Autoren zwischen 1770 und 1820; erscheint 1983 in edition suhrkamp: Hefte für kritische Literaturwissenschaft 5. Hg. v. Christa Bürger.

19 Vgl. u. a. Walter Raitz u. Erhard Schütz (Hg.): Der alte Kanon neu. Zur Revision des literarischen Kanons in Wissenschaft und Unterricht (Lesen 2) Opladen 1976; sowie Bodo Lecke (Hg.): Literatur der Klassik I u. II (projekt deutschunterricht 7 u. 9) Stuttgart 1974 u. 1975. - Zur neuesten , Kanone-Diskussion vgl. Rolf Geißler: Arbeit am literarischen Kanon. Perspektiven der Bürgerlichkeit. Paderborn 1982; ferner: Neue Lektüre klassischer Texte - Der Deutschunterricht 35 (1983) H. 1.

20 Dazu z. B. Hans Ch. Buch (Hg.): Von Goethe lernen? Fragen der Klassikrezeption (Literaturmagazin $2=$ dnb 49) Reinbek 1974, und Leo Kreutzer: Mein Gott Goethe. Essays (dnb 136) Reinbek 1980.

21 Heinz O. Burger ( $\mathrm{Hg}$.): Begriffsbestimmung der Klassik und des Klassischen (Wege der Forschung 210) Darmstadt 1972, dazu in neuerer Sicht Bodo Lecke: Literatur der deutschen Klassik. Rezeption und Wirkung. Heidelberg 1980, S. 35-50. - Vgl. als gleichzeitige und alternative Position Egidius Schmalzriedt: Inhumane Klassik. Vor- 
lesung wider ein Bildungsklischee. München 1971. Hier werden die Mißverständnisse zum Klassik-Begriff vor allem der Rezeptionsgeschichte dieser Kategorie angelastet, die einem geschichtlichen Verständnis des bezeichneten Phänomens entgegenwirkt.

22 Manfred Windfuhr: Kritik des Klassikbegriffs. In: Études Germaniques 29 (1974), S. 302-318. Hier S. 302.

23 Friedrich Sengle: Die klassische Kultur von Weimar, sozialgeschichtlich gesehen. In: IASL 3 (1978), S. 68-86. Hier S. 85.

$24 \mathrm{Vgl}$. als Beispiel für die Interpretation eines umfangreichen Textkorpus Karl O. Conrady (Hg.): Deutsche Literatur zur Zeit der Klassik. Stuttgart 1977.

$25 \mathrm{Vgl}$. hierzu für Goethe die grundlegenden Veröffentlichungen von Karl R. Mandelkow (s. Anm. 1 1); auf die Situation der jüngsten Vergangenheit bezogen ders. : Wandlungen des Klassikbildes in Deutschland im Lichte gegenwärtiger Klassikkritik. In Conrady: Literatur zur Zeit der Klassik, S. 423-439; ferner Mandelkow: Goethe im Urteil seiner Kritiker. Dokumente zur Wirkungsgeschichte Goethes in Deutschland. 1773-1918. 3 Bde. München 1975-1979. Dazu auch Paul Rilla: Goethe in der Literaturgeschichte. Zur Problematik der bürgerlichen Bildung. In P. R.: Literatur als Geschichte. Zwei Streitschriften. München 1978, S. 95-164; Bodo Lecke (Hg.): Goethe unter den Deutschen. Materialien zur literarischen Wirkung in 3 Jahrhunderten. Frankfurt a. M. u.a. 1978; ders.: Literatur der deutschen Klassik; Wolfgang Leppmann: Goethe und die Deutschen. Der Nachruhm eines Dichters im Wandel der Zeit und der Weltanschauungen. Ein Spiegelbild deutscher Kultur und Bildung. Bern u. München 1982 (erweiterte Auflage der Publikation von 1962).

26 Für den interdisziplinären Ansatz vgl. zur Romantik Richard Brinkmann (Hg.): Romantik in Deutschland. Ein interdisziplinäres Symposium. Stuttgart 1978 ( = Sonderband DVjs).

27 Das internationale Interesse an diesem Fragenkomplex zeigt sich in Heinz Rupp u. Hans-Gert Roloff $(\mathrm{Hg}$.) : Akten des 6. Internationalen Germanisten-Kongresses Basel 1980. Bd. 3. Bern u. a. 1980 (= Jb. f. Intern. Germanistik Reihe A, Bd. 8, 3); hier die Vorträge von J. M. Peck, D. van Abbé, H.-D. Dahnke, E. Ribbat (S. 319-343).

$28 \mathrm{Vgl}$. das Verfahren bei Dieter Borchmeyer: Die Weimarer Klassik. Eine Einführung. 2 Bde. (AT 2165/66) Königstein i. Ts. 1980.

29 So bei Brinkmann: Romantik in Deutschland, für die Romantik von 1790 bis 1830.

30 Vgl. dazu Fabian u. Schmidt-Biggemann: Das achtzehnte Jahrhundert; insbesondere die Aufsätze von W. Voßkamp (S. 53-69), R. Vierhaus (S. 71-86) und V. Lange (S. 87103).

31 Dazu Fritz Martini: Weimar - Mythos und Wirklichkeit. In: Goethe \& Co. Traum und Wirklichkeit der deutschen Klassik. Vorträge. Duisburg 1978, S. 43-53.

32 Stanley A. Corngold u. a. (Hg.): Aspekte der Goethezeit. Göttingen 1977.

33 Gerhart Hoffmeister (Hg.): Goethezeit: Studien zur Erkenntnis und Rezeption Goethes und seiner Zeitgenossen. Festschrift für Stuart Atkins. Bern u. München 1981.

34 Terence J. Reed: Die klassische Mitte. Goethe und Weimar 1775-1832 (Sprache und Literatur 115) Stuttgart 1982. (Engl. 1980).

35 Beispielsweise Claus Träger: Über Historizität und Normativität des Klassik-Begriffs. In: Weimarer Beiträge 25 (1979) H. 12, S. 5-20.

36 Zum Beispiel Helmut Brandt u. Manfred Beyer (Hg.): Ansichten der deutschen Klassik. Berlin 1981.

37 So Peter Weber u. a.: Kunstperiode. Studien zur deutschen Literatur des ausgehenden 18. Jahrhunderts. Berlin 1982.

38 Impulse. Aufsätze, Quellen, Berichte zur deutschen Klassik und Romantik. Hg. v. Walter Dietze u. Peter Goldammer. Berlin u. Weimar $1978 \mathrm{ff}$. 